\title{
Full-Rank Factoring of Elementary 2-Groups with Equal Size Factors
}

\section{Sándor Szabó}

Institute of Mathematics and Informatics, University of Pécs, Pécs, Hungary

Email:sszabo7@hotmail.com

How to cite this paper: Szabó, S. (2020) Full-Rank Factoring of Elementary 2-Groups with Equal Size Factors. Open Journal of Discrete Mathematics, 10, 89-95.

https://doi.org/10.4236/ojdm.2020.104009

Received: June 24, 2020

Accepted: September 13, 2020

Published: September 16, 2020

Copyright (c) 2020 by author(s) and Scientific Research Publishing Inc.

This work is licensed under the Creative Commons Attribution International License (CC BY 4.0).

http://creativecommons.org/licenses/by/4.0/

\begin{abstract}
In order to answer a question motivated by constructing substitution boxes in block ciphers we will exhibit an infinite family of full-rank factorizations of elementary 2-groups into two factors having equal sizes.
\end{abstract}

\section{Keywords}

Factorization of Finite Abelian Groups, Elementary 2-Groups, Full-Rank Subsets, Full-Rank Factorizations

\section{Introduction}

We will use multiplicative notation in connection with abelian groups. Let $G$ be a finite abelian group. The product $A_{1} \cdots A_{n}$ of the subsets $A_{1}, \ldots, A_{n}$ of $G$ is defined to be the set of the elements

$$
a_{1} \cdots a_{n}, \quad a_{1} \in A_{1}, \ldots, a_{n} \in A_{n} .
$$

The product $A_{1} \cdots A_{n}$ is called a direct product if

$$
a_{1} \cdots a_{n}=a_{1}^{\prime} \cdots a_{n}^{\prime}, \quad a_{1}, a_{1}^{\prime} \in A_{1}, \ldots, a_{n}, a_{n}^{\prime} \in A_{n}
$$

imply that $a_{1}=a_{1}^{\prime}, \ldots, a_{n}=a_{n}^{\prime}$. Let $B$ be a subset of $G$. If the product $A_{1} \cdots A_{n}$ is direct and it is equal to $B$, then we say that $B$ is factored into the subsets $A_{1}, \ldots, A_{n}$ or equivalently we say that the equation $B=A_{1} \cdots A_{n}$ is a factorization of $B$. In algebra books the most commonly occurring situation is when an entire abelian group is factored into a direct product of its subgroups.

The span of a subset $A$ of $G$ is the smallest subgroup of $G$ that contains $A$. The span of $A$ is denoted by $\langle A\rangle$. A subset $A$ of $G$ is called normalized if the identity element $e$ of $G$ is an element of $A$. If $A$ is a normalized subset of $G$ for which $\langle A\rangle=G$, then we say that $A$ is a full-rank subset of $G$. A factorization $G=A_{1} \cdots A_{n}$ is called a full-rank factorization if each factor is a full-rank subset of $G$.

Full-rank factorizations of finite abelian groups are intimately connected with the theory of error correcting, variable length codes and 
cryptography. For further details see for instance [1-4], respectively. Let $p$ be a prime. The direct product of $n$ isomorphic copies of a cyclic group of order $p$ is an abelian group and it is called an elementary $p$ group of rank $n$. In a letter Professor Claude Carlet asked me if there were full-rank factorizations of elementary 2 -groups into two factors of equal sizes [5]. He could use such a factorization for constructing substitution boxes, or S-boxes, in block ciphers.

The next few words try to explain how the S-box is built from a fullrank factorization. For more detail the reader should consult with [6]. Let $G=A_{1} A_{2}$ be a full-rank factorization of the elementary 2-group $G$ of rank $2 n$ such that $\left|A_{1}\right|=\left|A_{2}\right|=2^{n}$. Let $F_{2}$ be the finite Galois field with two elements and let $\pi_{1}: F_{2}^{n} \rightarrow A_{1}$ and $\pi_{2}: F_{2}^{n} \rightarrow A_{2}$ be bijective maps. Using $\pi_{1}$ and $\pi_{2}$ we define $F: F_{2}^{2 n} \rightarrow F_{2}^{2 n}$ by $F\left(\left(a_{1}, a_{2}\right)\right)=$ $\pi_{1}\left(a_{1}\right)+\pi_{2}\left(a_{2}\right)$. The fact that the factorization $G=A_{1} A_{2}$ is a full-rank factorization is a necessary condition that the S-box has a non-zero linearity. The non-linearity of the S-box is the desired property with cryptographic significance. (Problem 3 in Section 3 at the end of the paper is related to this issue.) The group theoretic argument we use to prove Theorem 1 does not give any useful hint how to increase the degree of non-linearity. It seems that more sophisticated techniques like polynomial type reasoning required.

In this note we will construct full-rank factorizations $G=A B$ of the elementary 2-group $G$ of rank $6 n$, where $|A|=|B|$ and $n \geq 3$.

\section{A Construction}

The main result of this paper is the following theorem.

Theorem 1. If $n \geq 3$, then the elementary 2-group of rank $6 n$ admits full-rank factorization into two factors of equal sizes.

Proof. Let $n$ be an integer such that $n \geq 3$. Let $G$ be an elementary 2 -group of rank $6 n$ with basis elements

$$
x_{1,1}, \ldots, x_{1,6}, \ldots, x_{n, 1}, \ldots, x_{n, 6} .
$$

Let

$$
\begin{aligned}
H_{i} & =\left\langle x_{i, 1}, x_{i, 2}, x_{i, 3}\right\rangle, \\
K_{i} & =\left\langle x_{i, 4}, x_{i, 5}, x_{i, 6}\right\rangle, \\
L_{i} & =\left\langle x_{i, 1}, \ldots, x_{i, 6}\right\rangle,
\end{aligned}
$$

for each $i, 1 \leq i \leq n$. It is clear that $\left|H_{i}\right|=\left|K_{i}\right|=2^{3}=8$. Further it is clear that the product $H_{i} K_{i}$ is direct and it is equal to $L_{i}$ for each $i, 1 \leq i \leq n$.

From the subgroup $H_{i}$ of $G$ we construct a subset $A_{i}$ of $G$ by removing and adding certain elements.

$\begin{array}{rr}\text { Remove : } & \text { Add : } \\ x_{i, 1} & x_{i, 1} x_{i, 4} \\ x_{i, 2} & x_{i, 2} x_{i, 5} \\ x_{i, 3} & x_{i, 3} x_{i, 6}\end{array}$

In other words we set

$$
A_{i}=\left(H_{i} \backslash\left\{x_{i, 1}, x_{i, 2}, x_{i, 3}\right\}\right) \cup\left\{x_{i, 1} x_{i, 4}, x_{i, 2} x_{i, 5}, x_{i, 3} x_{i, 6}\right\},
$$

for each $i, 1 \leq i \leq n$.

We claim that the product $A_{i} K_{i}$ is direct and it is equal to $L_{i}$ for each $i, 1 \leq i \leq n$. 
As the product $H_{i} K_{i}$ is direct and it is equal to $L_{i}$ it follows that the sets

$$
h_{i} K_{i}, \quad h_{i} \in H_{i}
$$

form a partition of $L_{i}$. We have constructed $A_{i}$ from $H_{i}$ by removing elements and adding elements. In the partition (1) we replace the set $x_{i, 1} K_{i}$ by the set $x_{i, 1} x_{i, 4} K_{i}$. Note that $x_{i, 4} K_{i}=K_{i}$ as $x_{i, 4} \in K_{i}$. In general,

$$
\begin{aligned}
x_{i, 1} K_{i} & =x_{i, 1} x_{i, 4} K_{i}, \\
x_{i, 2} K_{i} & =x_{i, 2} x_{i, 5} K_{i}, \\
x_{i, 3} K_{i} & =x_{i, 3} x_{i, 6} K_{i},
\end{aligned}
$$

and so the sets

$$
a_{i} K_{i}, \quad a_{i} \in A_{i}
$$

form a partition of $L_{i}$. The partition (2) is equivalent to that the product $A_{i} K_{i}$ is direct and it is equal to $L_{i}$, as required.

Let

$$
A=A_{1} \cdots A_{n}, \quad K=K_{1} \cdots K_{n} .
$$

We claim that the product $A K$ is direct and it is equal to $G$. Indeed,

$$
\begin{aligned}
G & =L_{1} \cdots L_{n} \\
& =\left(A_{1} K_{1}\right) \cdots\left(A_{n} K_{n}\right) \\
& =\left(A_{1} \cdots A_{n}\right)\left(K_{1} \cdots K_{n}\right) \\
& =A K .
\end{aligned}
$$

Thus the product $A K$ is direct and it is equal to $G$, as required. In particular the product $A_{1} \cdots A_{n}$ is direct and so $|A|=\left|A_{1}\right| \cdots\left|A_{n}\right|=$ $\left(2^{3}\right)^{n}=2^{3 n}$. In the above argument we used the observation that if the product $A_{i} K_{i}$ is direct and is equal to $L_{i}$ and if the product $L_{1} \cdots L_{n}$ is direct and is equal to $G$, then the product $A_{1} K_{1} \cdots A_{n} K_{n}$ is direct and is equal to $G$.

Next we claim that $\left\langle A_{i}\right\rangle=L_{i}$ for each $i, 1 \leq i \leq n$. As $x_{i, 2} x_{i, 3} \in A_{i}$ and $x_{i, 1} x_{i, 2} x_{i, 3} \in A_{i}$, it follows that $x_{i, 1} \in\left\langle A_{i}\right\rangle$. As $x_{i, 1} x_{i, 4} \in A_{i}$ and $x_{i, 1} \in\left\langle A_{i}\right\rangle$, it follows that $x_{i, 4} \in\left\langle A_{i}\right\rangle$. A similar reasoning gives that in general

$$
\begin{array}{lll}
x_{i, 1}, x_{i, 4} & \in & \left\langle A_{i}\right\rangle, \\
x_{i, 2}, x_{i, 5} & \in & \left\langle A_{i}\right\rangle, \\
x_{i, 3}, x_{i, 6} & \in & \left\langle A_{i}\right\rangle .
\end{array}
$$

Thus $\left\langle A_{i}\right\rangle=L_{i}$, as we claimed.

Since $e \in A_{1}, \ldots, e \in A_{n}$, we get that $A_{1}, \ldots, A_{n} \subset A_{1} \cdots A_{n}=A$. It follows that $\langle A\rangle=G$. In other words $A$ is a full-rank subset of $G$.

Let $f$ be the cyclic permutation of the numbers $1, \ldots, n$ defined by

$$
\left[\begin{array}{ccccc}
1 & 2 & \ldots & n-1 & n \\
f(1) & f(2) & \ldots & f(n-1) & f(n)
\end{array}\right]=\left[\begin{array}{ccccc}
1 & 2 & \ldots & n-1 & n \\
2 & 3 & \ldots & n & 1
\end{array}\right] .
$$

From the subgroup $K$ of $G$ we construct a subset $B$ of $G$. We do this by removing certain subsets from $K$ and adding certain subsets to $K$.

$$
\begin{array}{rr}
\text { Remove : } & \text { Add : } \\
x_{i, 4} K_{f(i)} & x_{i, 4} x_{f(i), 1} K_{f(i)} \\
x_{i, 5} K_{f(i)} & x_{i, 5} x_{f(i), 2} K_{f(i)} \\
x_{i, 6} K_{f(i)} & x_{i, 6} x_{f(i), 3} K_{f(i)}
\end{array}
$$

We claim that

$$
x_{i, 4} K_{f(i)} A=x_{i, 4} x_{f(i), 1} K_{f(i)} A,
$$




$$
\begin{aligned}
& x_{i, 5} K_{f(i)} A=x_{i, 5} x_{f(i), 2} K_{f(i)} A, \\
& x_{i, 6} K_{f(i)} A=x_{i, 6} x_{f(i), 3} K_{f(i)} A,
\end{aligned}
$$

for each $i, 1 \leq i \leq n$. There are $3 n$ equations to check. But the number of essentially distinct cases can be reduced to 3 . For the sake of definiteness we verify the first equation. We compute the left hand side and we compute the right hand side.

$$
\begin{aligned}
x_{i, 4} K_{f(i)} A & =x_{i, 4} K_{f(i)}\left(A_{1} \cdots A_{n}\right) \\
& =x_{i, 4}\left(K_{f(i)} A_{f(i)}\right) \bar{A}_{f(i)} \\
& =x_{i, 4} L_{f(i)} A_{f(i)}
\end{aligned}
$$

Here $\bar{A}_{f(i)}$ is computed in the following way. We delete $A_{f(i)}$ from the list $A_{1}, \ldots, A_{n}$ and multiply the remaining sets.

$$
\begin{aligned}
x_{i, 4} x_{f(i), 1} K_{f(i)} A & =x_{i, 4} x_{f(i), 1} K_{f(i)}\left(A_{1} \cdots A_{n}\right) \\
& =x_{i, 4} x_{f(i), 1}\left(K_{f(i)} A_{f(i)}\right) \bar{A}_{f(i)} \\
& =x_{i, 4} x_{f(i), 1} L_{f(i)} \bar{A}_{f(i)} \\
& =x_{i, 4} L_{f(i)} \bar{A}_{f(i)}
\end{aligned}
$$

The last step hinges on the fact that $x_{f(i), 1} \in L_{f(i)}$ and so $x_{f(i), 1} L_{f(i)}=L_{f(i)}$. The remaining cases can be settled in an analogous way.

We claim that the sets

$$
\begin{gathered}
x_{i, 4} K_{f(i)} A, \\
x_{i, 5} K_{f(i)} A, \\
x_{i, 6} K_{f(i)} A
\end{gathered}
$$

are pair-wise disjoint. This claim is of course the same as the claim that the sets

$$
\begin{aligned}
& x_{i, 4} x_{f(i), 1} K_{f(i)} A, \\
& x_{i, 5} x_{f(i), 2} K_{f(i)} A, \\
& x_{i, 6} x_{f(i), 3} K_{f(i)} A
\end{aligned}
$$

are pair-wise disjoint. There are $3 n$ sets and we claim that $\left(\begin{array}{c}3 n \\ 2\end{array}\right)$ pairs of sets are disjoint. However, the number of the essentially different cases is not more than $\left(\begin{array}{l}8 \\ 2\end{array}\right)=28$.

In order to prove the claim let us assume on the contrary that two distinct subsets are not disjoint. Among the many possible cases let us consider the following case first.

$$
x_{i, 4} K_{f(i)} A \cap x_{i, 5} K_{f(i)} A \neq \emptyset
$$

It follows that

$$
\begin{gathered}
x_{i, 4}\left(K_{f(i)} A_{f(i)}\right) \bar{A}_{f(i)} \cap x_{i, 5}\left(K_{f(i)} A_{f(i)}\right) \bar{A}_{f(i)} \neq \emptyset, \\
x_{i, 4} L_{f(i)} \bar{A}_{f(i)} \cap x_{i, 5} L_{f(i)} \bar{A}_{f(i)} \neq \emptyset .
\end{gathered}
$$

For the sake of definiteness suppose that $f(i)=1$. Consequently, $i=n$ and

$$
x_{n, 4} L_{1} \bar{A}_{1} \cap x_{n, 5} L_{1} \bar{A}_{1} \neq \emptyset .
$$


There are elements $l_{1}, l_{1}^{\prime} \in L_{1}$ and

$$
a_{1}, a_{1}^{\prime} \in A_{1}, \ldots, a_{n}, a_{n}^{\prime} \in A_{n}
$$

such that

$$
l_{1} a_{2} \cdots a_{n} x_{n, 4}=l_{1}^{\prime} a_{2}^{\prime} \cdots a_{n}^{\prime} x_{n, 5} .
$$

Using the fact that the product $L_{1} \cdots L_{n}$ is direct and that

$$
A_{1} \subset L_{1}, \ldots, A_{n} \subset L_{n}
$$

we get that

$$
l_{1}=l_{1}^{\prime}, a_{2}=a_{2}^{\prime}, \ldots, a_{n-1}=a_{n-1}^{\prime}, a_{n} x_{n, 4}=a_{n}^{\prime} x_{n, 5} .
$$

The last equation means that $A_{n} x_{n, 4} \cap A_{n} x_{n, 5} \neq \emptyset$. On the other hand after listing the elements of $A_{n} x_{n, 4}$ and $A_{n} x_{n, 5}$ a routine inspection reveals that $A_{n} x_{n, 4} \cap A_{n} x_{n, 5}=\emptyset$. The details of the inspection are listed in Table 1 . Let us consider another case among the many possibilities.

Table 1. The elements of the subsets $H_{n}, A_{n}, A_{n} x_{n, 4}, A_{n} x_{n, 5}$.

\begin{tabular}{cccc}
\hline$H_{n}$ & $A_{n}$ & $A_{n} x_{n, 4}$ & $A_{n} x_{n, 5}$ \\
\hline$e$ & $e$ & $x_{n, 4}$ & $x_{n, 5}$ \\
$x_{n, 1}$ & $x_{n, 1} x_{n, 4}$ & $x_{n, 1}$ & $x_{n, 1} x_{n, 4} x_{n, 5}$ \\
$x_{n, 2}$ & $x_{n, 2} x_{n, 5}$ & $x_{n, 2} x_{n, 5} x_{n, 4}$ & $x_{n, 2}$ \\
$x_{n, 3}$ & $x_{n, 3} x_{n, 6}$ & $x_{n, 3} x_{n, 6} x_{n, 4}$ & $x_{n, 3} x_{n, 6} x_{n, 5}$ \\
$x_{n, 1} x_{n, 2}$ & $x_{n, 1} x_{n, 2}$ & $x_{n, 1} x_{n, 2} x_{n, 4}$ & $x_{n, 1} x_{n, 2} x_{n, 5}$ \\
$x_{n, 1} x_{n, 3}$ & $x_{n, 1} x_{n, 3}$ & $x_{n, 1} x_{n, 3} x_{n, 4}$ & $x_{n, 1} x_{n, 3} x_{n, 5}$ \\
$x_{n, 2} x_{n, 3}$ & $x_{n, 2} x_{n, 3}$ & $x_{n, 2} x_{n, 3} x_{n, 4}$ & $x_{n, 2} x_{n, 3} x_{n, 5}$ \\
$x_{n, 1} x_{n, 2} x_{n, 3}$ & $x_{n, 1} x_{n, 2} x_{n, 3}$ & $x_{n, 1} x_{n, 2} x_{n, 3} x_{n, 4}$ & $x_{n, 1} x_{n, 2} x_{n, 3} x_{n, 5}$ \\
\hline
\end{tabular}

$$
x_{i, 4} K_{f(i)} A \cap x_{j, 4} K_{f(j)} A \neq \emptyset
$$

It follows that

$$
x_{i, 4} L_{f(i)} \bar{A}_{f(i)} \cap x_{j, 4} L_{f(j)} \bar{A}_{f(j)} \neq \emptyset .
$$

In order to avoid unnecessary notational difficulties let us suppose that $f(i)=1, i=n, f(j)=3, j=2$. Now

$$
x_{n, 4} L_{1} \bar{A}_{1} \cap x_{2,4} L_{3} \bar{A}_{3} \neq \emptyset .
$$

There are elements $l_{1} \in L_{1}, l_{3}^{\prime} \in L_{3}$ and (6) such that

$$
l_{1} a_{2} \cdots a_{n} x_{n, 4}=l_{3}^{\prime} a_{1}^{\prime} a_{2}^{\prime} a_{4}^{\prime} \cdots a_{n}^{\prime} x_{2,4} .
$$

It follows that

$l_{1}=a_{1}^{\prime}, a_{2}=a_{2}^{\prime} x_{2,4}, a_{3}=l_{3}^{\prime}, a_{4}=a_{4}^{\prime}, \ldots, a_{n-1}=a_{n-1}^{\prime}, a_{n} x_{n, 4}=a_{n}^{\prime}$

The last equation means that $A_{n} x_{n, 4} \cap A_{n} \neq \emptyset$. On the other hand after listing the elements of $A_{n} x_{n, 4}$ and $A_{n}$ a routine inspection reveals that $A_{n} x_{n, 4} \cap A_{n}=\emptyset$.

A similar argument can be used in connection with all the remaining cases. 
We claim that the product $A B$ is direct and it is equal to $G$. In order to verify the claim note that the sets

$$
A k, \quad k \in K
$$

form a partition of $G$. We have constructed $B$ from $K$ by replacing certain subsets $A k$ by certain subsets $A b$. Using the Equations (3), (4), (5) we can see that the sets

$$
A b, \quad b \in B
$$

form a partition of $G$. Partition (8) simply means that the product $A B$ is direct and it is equal to $G$, as required.

From the above result it follows that $|B|=|K|=\left|K_{1}\right| \cdots\left|K_{n}\right|=$ $2^{3 n}$. The point we would like to stress is that $|A|=|B|$ holds.

We claim that $\langle B\rangle=G$. Since $x_{i, 5} x_{i, 6} \in B$ and $x_{i, 4} x_{i, 5} x_{i, 6} \in B$, it follows that $x_{i, 4} \in\langle B\rangle$. As $x_{i, 4} \in\langle B\rangle$ and $x_{i, 4} x_{f(i), 1} \in B$, it follows that $x_{f(i), 1} \in\langle B\rangle$. In general

$$
\begin{aligned}
& x_{i, 4}, x_{f(i), 1} \in\langle B\rangle, \\
& x_{i, 5}, x_{f(i), 2} \in\langle B\rangle, \\
& x_{i, 6}, x_{f(i), 3} \in\langle B\rangle,
\end{aligned}
$$

for each $i, 1 \leq i \leq n$. Thus $\langle B\rangle=G$, as required. In other words $B$ is a full-rank subset of $G$.

\section{Open Problems}

We close the paper with a number of open problems. The smallest elementary 2-group $G$ for which the construction of the paper works has $2^{18}$ elements and so the factors $A$ and $B$ have $2^{9}$ elements. The word length in the commonly used computers is a power of 2 . Professor C. Carlet has advanced the following problem.

Problem 1. Is there a full-rank factoring $G=A B$ of the elementary 2 -group $G$ of order $2^{16}$ with $|A|=|B|$ ?

Here is a more ambitious problem.

Problem 2. Determine the minimum order of all elementary abelian 2-groups that admit full-rank factorizations with equal size factors.

In cryptography not the full-rank property of the factors is the key concept but rather the non-linearity of the factors.

Problem 3. In a factorization $G=A B$ of an elementary 2-group with $|A|=|B|$ try to maximize the deviation of the factors from linearity.

The next questions are motivated by pure group theoretical curiosity.

Problem 4. Can a finite abelian 2-group be factored into more than two full-rank factors of equal size?

Problem 5. Can a finite abelian p-group be factored into full-rank factors of equal size? 


\section{Conflicts of Interest}

The author declares no conflicts of interest regarding the publication of this paper.

\section{References}

[1] Dinitz, M. (2006) Full Rank Tilings of Finite Abelian Groups. SIAM Journal on Discrete Mathematics, 20, 160-170. https://doi.org/10.1137/S0895480104445794

[2] Etzion, T. and Vardy, A. (1998) On Perfect Codes and Tilings: Problems and Solutions. SIAM Journal on Discrete Mathematics, 11, 205-223. https://doi.org/10.1137/S0895480196309171

[3] Qu, M.H. and Venstone, S.A. (1994) Factorizations in the Elementary Abelian $p$-Groups and Their Cryptographic Significance. Journal of Cryptography, 7, 201-212. https://doi.org/10.1007/BF00203963

[4] Szabó, S. (2006) Completing Codes and the Rédei Property of Groups. Theoretical Computer Science, 359, 449-454. https://doi.org/10.1016/j.tcs.2006.02.002

[5] Carlet, C. and Youssef, A. Building S-Boxes from Full-Rank Factorization of an Elementary 2-Group. Private Communication.

[6] Carlet, C. (2010) Vectorial Boolean Functions for Cryptography. In: Crama, Y., Ed., Boolean Models and Methods in Mathematics, Computer Science, and Engineering, Cambridge University Press, Cambridge, 398-470.

https://doi.org/10.1017/CBO9780511780448.012 